\title{
AEM / HRTEM Studies of Interface Diffusion-Controlled Transformations
}

\author{
I. G., Solórzano. \\ Department of Materials Science and Metallurgy, PUC-Rio, Rio de Janeiro, Brazil.
}

Interface diffusion plays a central and rate-determining role in a variety of solid phase transformations. In polycrystalline materials, high angle grain boundaries are the prominent interfaces, acting as high diffusion paths for the transport of solute atoms. As a result, a number of well-defined microstructures are developed, depending upon the material's composition and treatment conditions. From the phenomenological point of view, it is common to consider two major categories: transformations driving by stationary grain boundaries and those driven by moving grain boundaries. The former corresponds to classical heterogeneous precipitation and segregation phenomena, and the latter is related to discontinuous precipitation, coarsening and dissolution reactions in materials. In the present contribution it is shown, by means of a representative example, that the application of analytical (AEM) and highresolution transmission electron microscopy (HRTEM) allows one to obtain, respectively, nano-scale compositional together with structural information down to the atomic level.

The example presented here refers to the discontinuous precipitation (DP) phenomenon taking place in an Al-15 at \% Ag alloy upon isothermal aging treatments. As shown in the micrograph of fig. 1a, DP results from the decomposition of a supersaturated solid solution $\alpha^{0}$ in a structural identical, but solute depleted phase $\alpha^{\prime}$ and a $\beta$ equilibrium precipitate behind an advancing grain boundaries, developing a lamellar structure. Extended aging treatment, under steady-state growth conditions, as reflected by the regular inter-lamellar space, promotes the mutual impingement of different reaction fronts, as shown on fig. $1 b$. Interesting of noticing in this system is a the thickening of $\beta$ lamellae by lateral grow through a ledge mechanism as shown on fig. 2. In this micrograph it is also observed the transformation takes place with the generation of dislocations in the $\alpha$ ' lamellae. HRTEM indeed collaborates the layer-bylayer thickening of $\beta$ preserving a coherent interface with $\alpha^{\prime}$ in order to minimize surfaces energy, as shown in fig. 3. The orientation relationship corresponds to $\{0001\}_{\beta} / /\{111\}_{\alpha},<1120>_{\beta} / /<110>_{\alpha}$. The theoretical solution of the diffusion equation predicts a solute profile "frozen-in, behind the boundary reaction front in the product $\alpha^{\prime}$ lamellae ${ }^{1}$. The experimental proof, however, requires the use of highresolution microanalysis, as recently reviewed ${ }^{2}$. On the other hand, real $\alpha^{0} / \alpha^{\prime}$ interface shapes show concave-forward curvature, as the one presenting on fig. $4 \mathrm{~b}$, allowing to expect the composition at the $\alpha / \beta$ interface to be capillarity-modified with respect to the equilibrium $\mathrm{X}_{\mathrm{e}}$. Indeed, local composition distribution in $\alpha$ lamellae is possible to measure with dedicated FEG STEM, stopping $1 \mathrm{~nm}$ probes at 20 $\mathrm{nm}$ intervals and the EDS spectra analyzed. Fig. 4a shows that the micro analytical results generate a $\mathrm{Ag}$ profile at the advancing edge of the central $\alpha$ ' lamellae shown in fig. 4b. Optimal experimental conditions have met in this example since the $\beta$ precipitates have been preferentially etched out to permit the analysis in $\alpha^{\prime}$ close to the $\alpha^{\prime} / \beta$ interface while the sample was conveniently oriented with respect to the incident electron beam so that the foil edge remains perpendicular to the $\mathrm{x}$-ray path towards the EDS detector, thereby enabling local composition measurements along the same foil thickness with minimum $\mathrm{x}$-ray absorption. This procedure is reproducible and invites to be applied to other alloy systems presenting DP and other interface diffusion-controlled reactions ${ }^{3}$.

\section{References}

[1] J.W. Cahn, Acta Metall, 7, 18, (1959

[2] P. Zieba and W. Gust, Int. Mater. Rev., 43, 70, (1998)

[3] This work has been sponsored by CNPq (Brazil). 

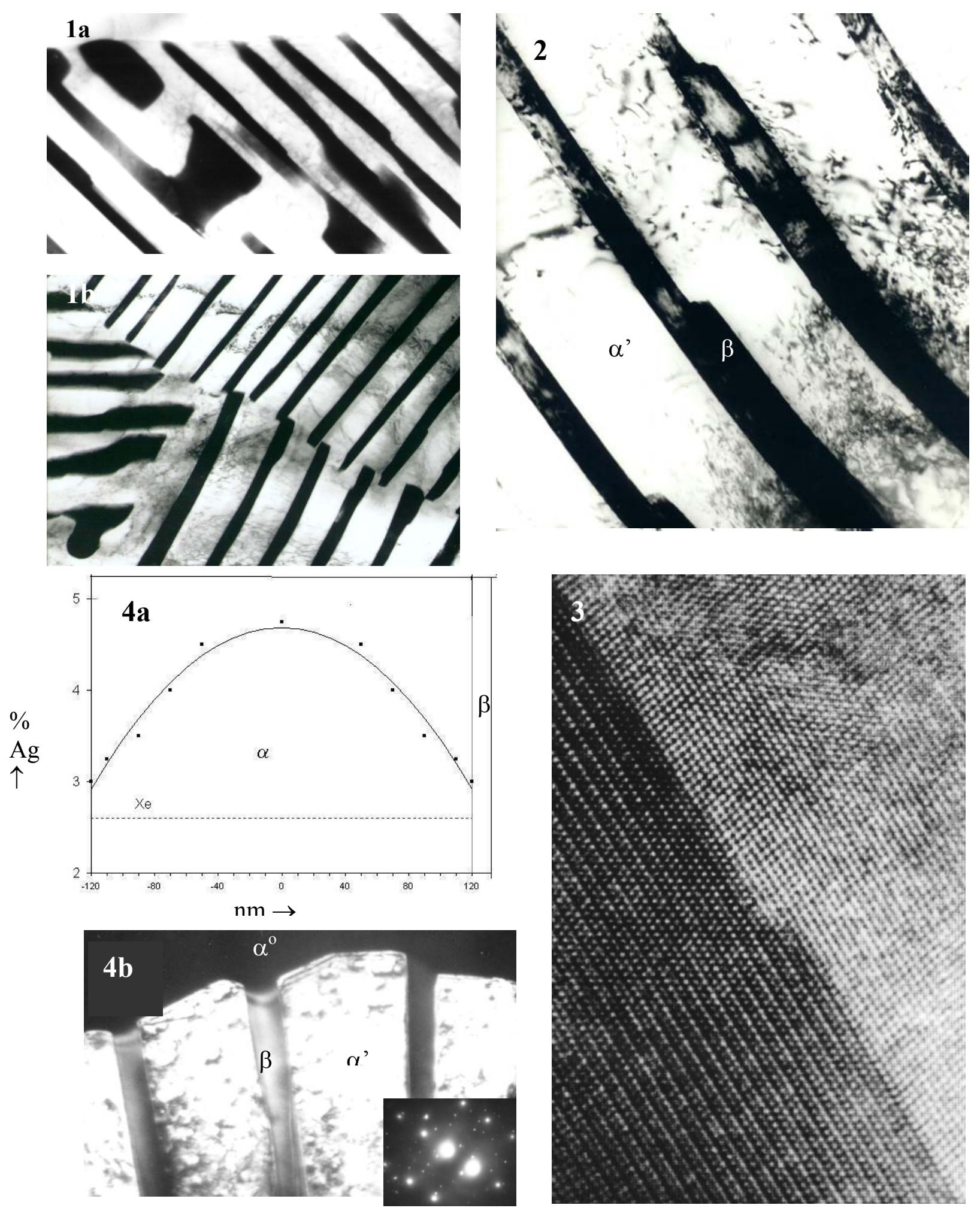

FIG. 1- Bright field TEM images of a) DP reaction front and b) the result of the impingement of 3 reaction fronts, all with a regular lamellar structure.

FIG. 2 - Thickening of precipitate lamellae by lateral growth upon prolonged aging.

FIG. 3 - HREM of the $\alpha / \beta$ coherent interface with atomic- scale ledge.

FIG. 4 - a) Compositional profile measured at the advancing edge of the central lamella shown in 4b) together with the selected area diffraction pattern. 